\title{
Failure to demonstrate specificity of the morphological and histochemical changes in mucosa adjacent to colonic carcinoma (transitional mucosa)
}

\author{
P. ISAACSON AND P. R. A. ATTWOOD \\ From the Department of Pathology, Southampton University Hospital, Southampton SO9 4XY, UK
}

SUMMARY Characteristic morphological and histochemical changes in mucosa adjacent to colorectal adenocarcinomas have been described. It has been suggested that this type of mucosa, labelled transitional mucosa (TM) because of its specific association with colorectal adenocarcinoma, is indicative of a premalignant change. In an investigation of mucosa adjacent to anal tumours extending into the rectum and mucosa from solitary ulcer syndrome and colostomies the mucosal alterations described in TM could be demonstrated. Thus TM is not specifically related to colorectal adenocarcinoma and probably arises as a secondary phenomenon. The claim that TM represents a premalignant change cannot be substantiated.

The term transitional mucosa was first used by Filipe in 1969 to describe mucosa adjacent to the edge of colonic adenocarcinoma which does not show any microscopic features of malignancy but within which a consistent alteration of mucin histochemistry can easily be demonstrated using the high iron diamine/alcian blue (HID/AB) stain. While the mucin in normal colonic mucosa is predominantly sulphated (brown/black with HID/AB stain), that in transitional mucosa is predominantly non-sulphated sialomucin (blue with HID/AB stain). Since the original report of Filipe these changes have been confirmed many times (Filipe, 1972; Filipe and Branfoot, 1974; Filipe and Cooke, 1974; Dawson and Filipe, 1976; Riddell and Levin, 1977) and characteristic morphological changes in transitional mucosa at both light and electron microscope level have been described (Dawson and Filipe, 1976; Riddell and Levin, 1977; Saffos and Rhatigan, 1977). These reports have led to the suggestion that the changes in transitional mucosa represent a specific premalignant alteration in colonic mucosa, perhaps reflecting a reversion to a more primitive state. The possibility that the changes in transitional mucosa are a secondary rather than a primary phenomenon has been considered but rejected on the basis that these changes have not been found in association with any other condition.

Primary malignant tumours of the anus (squa-

Received for publication 18 September 1978 mous cell carcinoma and melanoma), when they extend into the rectum, may mimic rectal adenocarcinoma macroscopically but the rectal margin of these tumours should not show the morphological or histochemical changes of transitional mucosa if the condition is indeed a primary phenomenon. Similarly, although the microscopic appearances described in transitional mucosa resemble those described in solitary ulcer syndrome and other conditions associated with mucosal prolapse (for example, colostomy) (Rutter and Riddell, 1975), the mucin histochemistry in these conditions should be normal. Accordingly, we have compared the microscopic and histochemical features of transitional mucosa adjacent to colorectal carcinoma with the mucosa bordering anal melanomas and squamous cell carcinomas, solitary ulcer syndrome mucosa, and mucosa covering colostomies. Both the morphological and histochemical changes described as specific for transitional mucosa were present in these three conditions, suggesting that transitional mucosa is a secondary phenomenon.

\section{Material and methods}

The high iron diamine/alcian blue stain (Spicer, 1965) was used to examine 80 sections of colorectal adenocarcinoma with adjacent benign mucosa, 22 sections of normal rectosigmoid mucosa, and the tumour-mucosal junction of three anal melanomas, five anal squamous cell carcinomas, and one malignant lymphoma of the colon. In addition, 
rectal mucosa from nine cases of the solitary ulcer syndrome and mucosa from four colostomies was similarly studied. In all cases an adjacent section was stained with haematoxylin and eosin.

Suitable $\mathbf{H} \&$ E stained slides representative of each condition were examined using an eyepiece micrometer, and an average of 10 measurements of crypt depth and goblet cell height was obtained in each case and compared to the findings in normal mucosa from the rectosigmoid.

\section{Results}

The results are summarised in Tables 1,2 , and 3.

Table 1 Presence of histochemical changes described in transitional mucosa using the HID/AB stain ( $p H$ 2.5)

\begin{tabular}{lcccc}
\hline Mucosa & Total No. & \multicolumn{3}{c}{ Histological changes } \\
\cline { 2 - 5 } & & Pos. & Neg. & $\%$ Pos. \\
\hline $\begin{array}{l}\text { Adjacent to } \\
\text { adenocarcinoma }\end{array}$ & 80 & 80 & 0 & 100 \\
$\begin{array}{l}\text { Adjacent to } \\
\text { other carcinomas }\end{array}$ & 9 & 8 & 1 & 89.9 \\
$\begin{array}{l}\text { Solitary ulcer } \\
\text { Colostomy }\end{array}$ & 9 & 8 & 1 & 89.9 \\
\hline
\end{tabular}

Table 2 Crypt depth of colonic mucosa

\begin{tabular}{lcll}
\hline Mucosa & No. & Range (mm.) & Av. (mm.) \\
\hline $\begin{array}{l}\text { Normal } \\
\text { Adjacent to }\end{array}$ & 22 & $0.41-0.66$ & 0.54 \\
$\quad$ adenocarcinoma & 24 & $0.92-1.41$ & $1 \cdot 17$ \\
$\begin{array}{l}\text { Adjacent to } \\
\quad \text { other carcinomas }\end{array}$ & 5 & $0.99-1.23$ & 1.13 \\
$\begin{array}{l}\text { Solitary ulcer } \\
\text { Colostomy }\end{array}$ & 7 & $0.73-1.30$ & 1.01 \\
\hline
\end{tabular}

Table 3 Goblet cell height in colonic mucosa

\begin{tabular}{lcll}
\hline Mucosa & No. & Range $(\mu)$ & Av. $(\mu)$ \\
\hline Normal & 21 & $25-37$ & 29 \\
Adjacent to & 27 & $33-57$ & 47 \\
$\quad$ adenocarcinoma & & $36-57$ & 46 \\
$\begin{array}{l}\text { Adjacent to } \\
\text { other carcinomas }\end{array}$ & 7 & & \\
Solitary ulcer & 8 & $29-49$ & 40 \\
Colostomy & 4 & $32-46$ & 37 \\
\hline
\end{tabular}

Normal rectosigmoid mucosa (Figs 1 and 2) contained predominantly sulphomucins which stained brown/black, a few cells containing sialomucin staining blue towards the surface. In contrast, the mucosa adjacent to colorectal adenocarcinomas (transitional mucosa) was thicker, the crypts being elongated and the individual goblet cells taller (Fig. 1). With the HID/AB stain sialomucins pre- dominated (Fig. 2). With rare exceptions mucosa adjacent to other tumours, from solitary ulcer syndrome and from colostomies (Figs 1 and 2), showed similar morphological and histochemical changes. Measured crypt depth and goblet cell height were increased in transitional mucosa and in the other conditions studied (Tables 2 and 3).

\section{Discussion}

Our results suggest that the morphological appearances and histochemical characteristics of transitional mucosa do not reflect a specific premalignant change but are secondary to the presence of colonic adenocarcinoma. The mucosal changes in solitary ulcer syndrome, in common with those in colostomy mucosa, are thought to be due to prolapse since they are seen to a variable degree in any condition in which there is prolapse (Rutter and Riddell, 1975). The exact mechanism is not understood although alteration in submucosal blood supply is one possible explanation. In fully developed solitary ulcer syndrome the increased height of mucosal crypts is accompanied by lamina propria fibrosis and proliferation of smooth muscle into the lamina propria; these changes, perhaps reflecting a severe degree of prolapse, are rarely seen in colostomy mucosa, and we have not observed them adjacent to adenocarcinomas. Prolapsing forces are to be expected at the edge of mucosal tumours and could well account for the appearances of transitional mucosa. Moreover, the finding that the zone of transitional mucosa is larger in more advanced tumours (Filipe and Branfoot, 1974) is consistent with this view since the edges of these tumours tend to project into the lumen of the colon more than in early smaller lesions. This relationship between the size of the zone of transitional mucosa and the stage of the carcinoma is exactly the opposite to that between the colorectal adenoma, a well-recognised premalignant lesion, and adenocarcinoma (Muto et al., 1975). The decreasing frequency and size of residual adenomatous mucosa with advancing tumour stage is one of the more convincing arguments cited in favour of the premalignancy of adenomas.

Ultrastructural studies (Dawson and Filipe, 1976) have shown that in transitional mucosa there is persistence of immature and intermediate cells at higher levels of the crypt than in normal mucosa and that these cells are shed into the crypt prematurely. To us this suggests a regenerative phenomenon rather than a pre-neoplastic change and could explain the occasional finding of transitional mucosa remote from carcinomas.

The possibility that transitional mucosa is a 

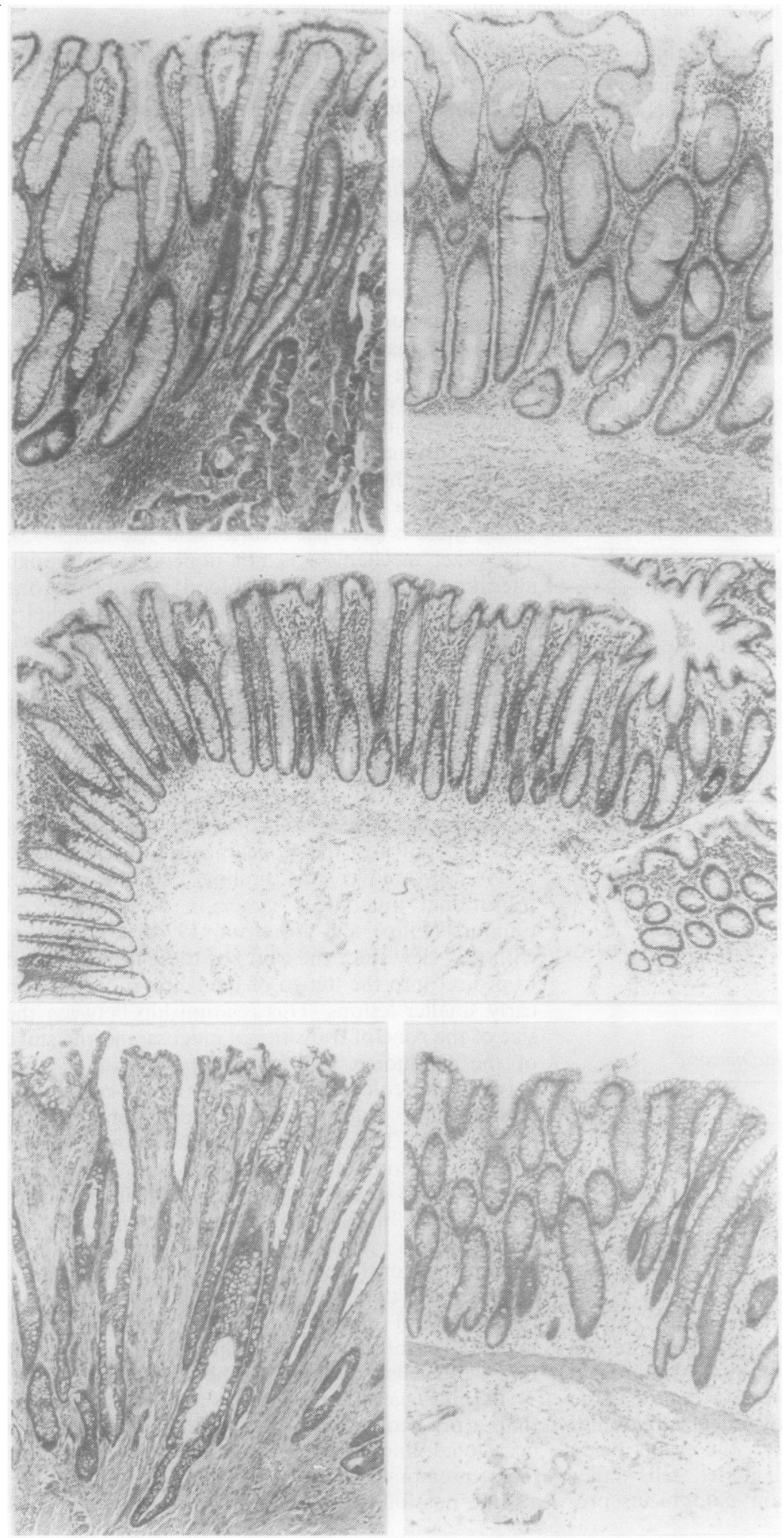

Fig. 1 In contrast to normal mucosa (centre) mucosa adjacent to an adenocarcinoma (top left) and anal melanoma (top right) show deeper crypts with some branching and larger goblet cells. Similar changes are apparent in mucosa from solitary ulcer. syndrome (bottom left) and from colostomy (bottom right). Haematoxylin and eosin $\times 40$ 

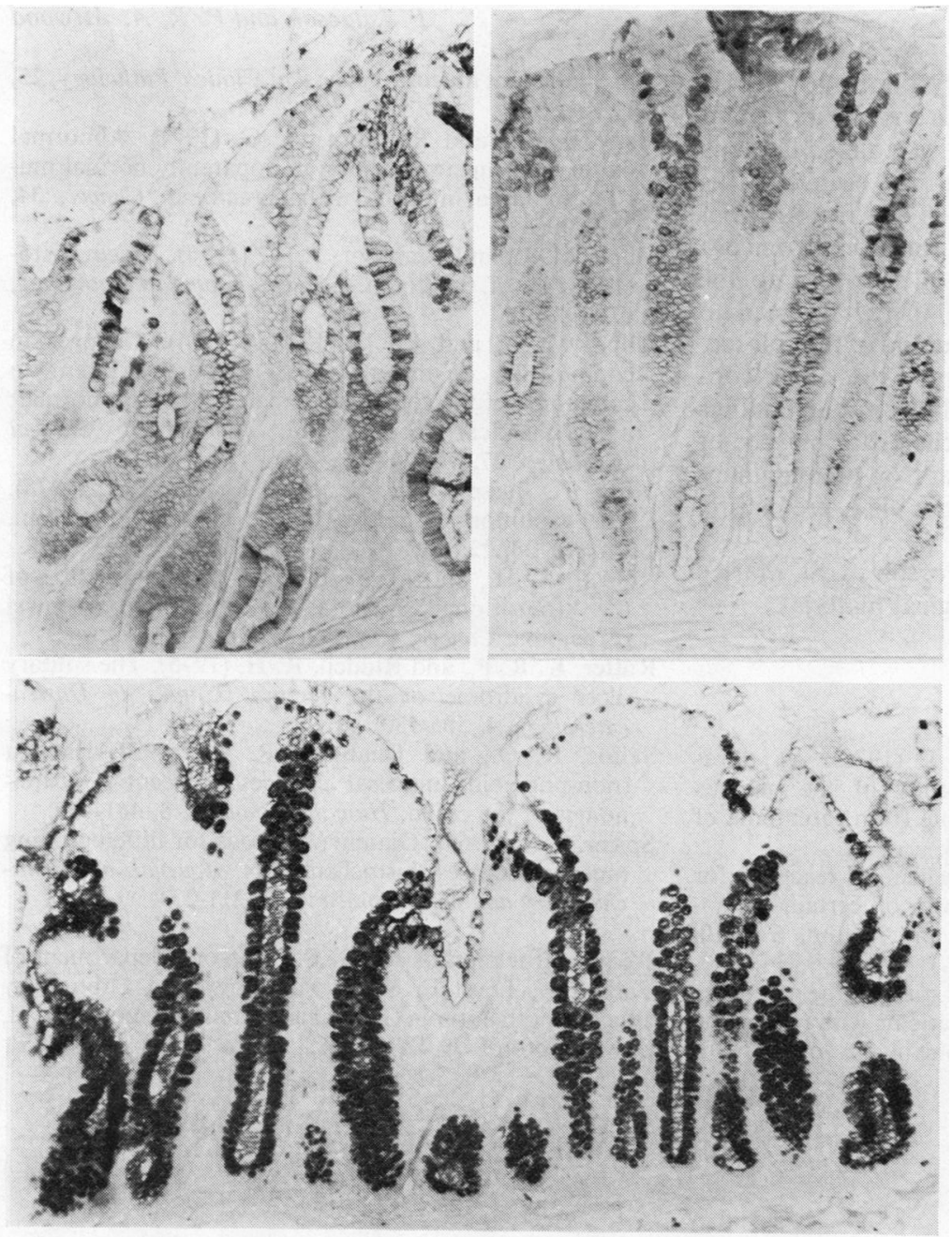

Fig. 2 In normal mucosa (centre) predominating sulphomucins stain dark brown while in mucosa adjacent to adenocarcinoma (top left) and anal melanoma (top right) blue staining sialomucins predominate. Similar histochemical alteration is apparent in mucosa from solitary ulcer syndrome (bottom left) and colostomy (bottom right). High iron diamine/alcian blue at pH 2.5. With blue filter $\times 100$

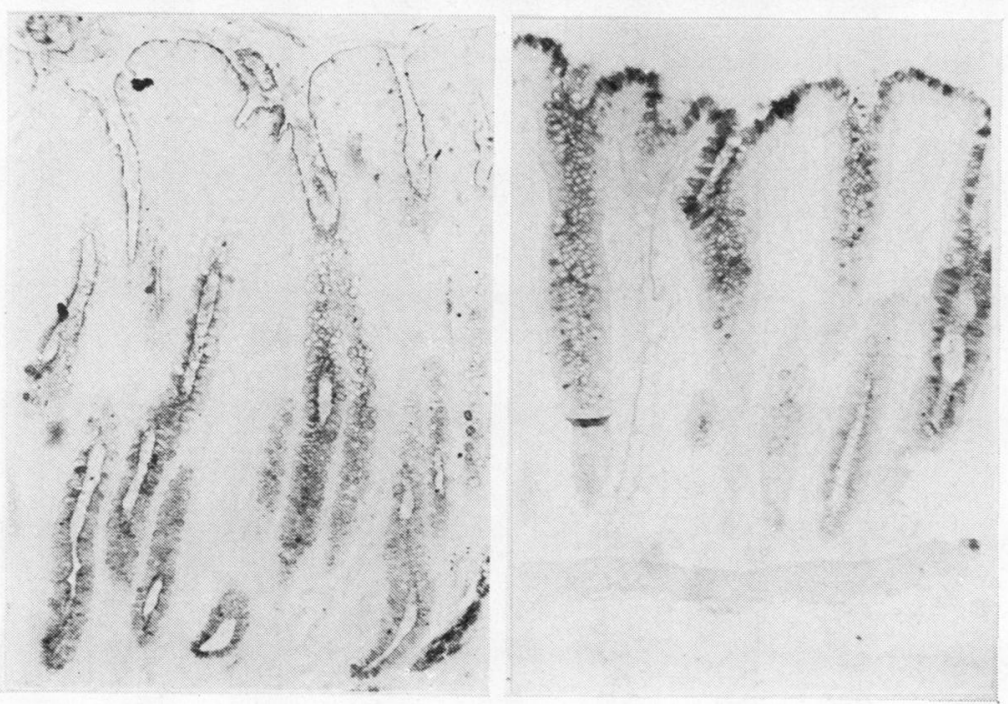


secondary rather than a primary phenomenon has been considered (Filipe, 1969) but the failure to find this type of mucosa in ulcerative colitis, Crohn's disease, and diverticular disease has been cited as evidence against this possibility. Apart from a brief mention of the absence of transitional mucosa adjacent to secondary tumours ulcerating into the colon (Filipe and Branfoot, 1976) there have been no studies of mucosa adjacent to tumours of the colorectum other than carcinoma nor of the other conditions we have studied. In the light of our findings the suggestion that mucin histochemistry may be of value in detecting early malignant or premalignant change in colonic biopsies is, in our view, premature.

We are indebted to Dr B. C. Morson of St Mark's Hospital for the loan of histological material.

\section{References}

Dawson, P. A., and Filipe, M. I. (1976). An ultrastructural and histochemical study of the mucous membrane adjacent to and remote from carcinoma of the colon. Cancer, 37, 2388-2398.

Filipe, M. I. (1969). Value of histochemical reactions for muco-substances in the diagnosis of certain pathological conditions of the colon and rectum. Gut, 10, 577-586.

Filipe, M. I. (1972). The value of a study of the mucosubstances in rectal biopsies from patients with carcinoma of the rectum and lower sigmoid in the diagnosis of premalignant mucosa. Journal of Clinical Pathology, 25, 123-128.

Filipe, M. I., and Branfoot, A. C. (1974). Abnormal patterns of mucus secretion in apparently normal mucosa of large intestine with carcinoma. Cancer, 34, 282-290.

Filipe, M. I., and Branfoot, A. C. (1976). Mucin histochemistry of the colon. Current Topics in Pathology, 63, 143-178.

Filipe, M. I., and Cooke, K. B. (1974). Changes in composition of mucin in the mucosa adjacent to carcinoma of the colon as compared with the normal: a biochemical investigation. Journal of Clinical Pathology, 27, 315-318.

Muto, T., Bussey, H. J. R., and Morson, B. C. (1975). The evolution of cancer of the colon and rectum. Cancer, 36, 2251-2270.

Riddell, R. H., and Levin, B. (1977). Ultrastructure of the "transitional" mucosa adjacent to large bowel carcinoma. Cancer, 40, 2509-2522.

Rutter, K. R. P., and Riddell, R. H. (1975). The solitary ulcer syndrome of the rectum. Clinics in Gastroenterology, 4, 505-530.

Saffos, R. O., and Rhatigan, R. M. (1977). Benign (non-polypoid) mucosal changes adjacent to carcinomas of the colon. Human Pathology, 8, 441-449.

Spicer, S. S. (1965). Diamine methods for differentiating muco-substances histochemically. Journal of Histochemistry and Cytochemistry, 13, 211-234.

Requests for reprints to: Dr P. Isaacson, Department of Pathology, Level E, South Pathology and Laboratory Block, Southampton General Hospital, Tremona Road, Southampton SO9 4XY, UK. 